\title{
Aspects écologiques des travaux d'aménagement des cours d'eau ORIENTATIONS ET PRINCIPES GÉNÉRAUX *
}

\author{
PAR
}

\author{
J. Verneaux J.-P. Vergon ET M. Larinier \\ Laboratoire d'Hydroécologie du C.T.G.R.E.F., Paris \\ et Centre d'Hydrobiologie de l'Université \\ de Besançon
}

\section{1 - Motivation}

Lorsque l'écologiste tente, par une analyse objective, de définir les raisons scientifiques présidant aux travaux dits «d'aménagement» effectués sur les systèmes d'eau courante, il ne trouve, en dehors de cas exceptionnels, qu'un nombre très restreint de motifs techniques fragmentaires. Evoquant la nécessité de limiter les inondations dues aux crues et l'éventuelle récupération de terres susceptibles d'être cultivées, les travaux se traduisent généralement par des redressements et des recalibrages destinés à assurer l'évacuation la plus rapide possible des eaux des points hauts vers les points bas.

A cette conception rudimentaire et dangereuse qui, transformant les systèmes aquatiques en chenaux d'évacuation, s'oppose à une politique cohérente des ressources aquatiques, s'ajoute le fait que les techniques utilisées ne peuvent assurer la pérennité des travaux effectués et des ouvrages construits. Les pratiques actuelles les plus fréquentes lèsent plus ou moins gravement l'écosystème « eau courante », détruit avant que d'être connu, et compromettent la qualité même des eaux, étroitement tributaire de la structure de l'écosystème. L'entreprise d'interventions chirurgicales suppose une connaissance préalable suffisante de l'anatomie et de la physiologie de l'organisme considéré, en l'occurrence l'écosystème « eau courante».

\section{2 - L'écosystème "eau courante"}

Cette entité écologique apparaît comme l'ensemble intégré du milieu (dissocié en composantes ou paramètres) et des peuplements associés (espèces vivantes), comme l'unité fonctionnelle résultant de l'ensemble des relations (flèches du tableau ci-dessous) existant entre espèces, entre paramètres, puis entre espèces et milieu.

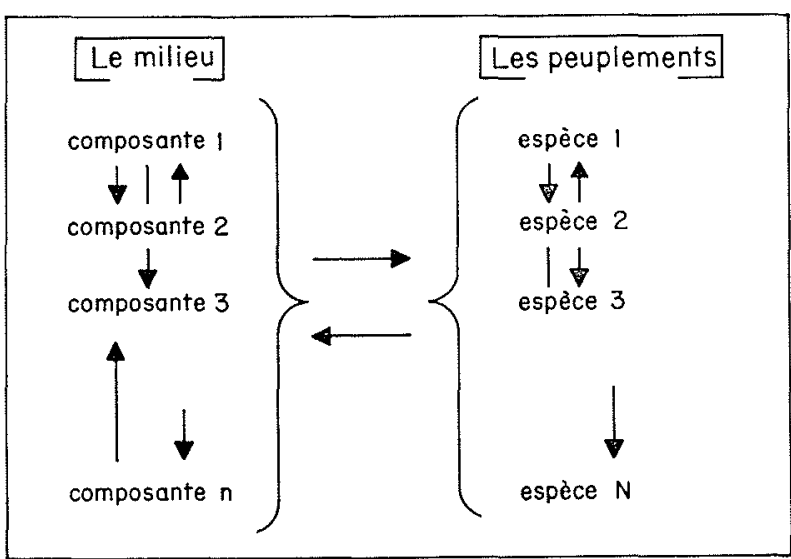

L'écosystème : interrelations

Les éléments globaux de l'écosystème : l'eau, le substrat (lit-berges-nappe) et l'édifice trophique, sont fonctionnellement indissociables et toute modification de l'un des éléments se répercute au niveau des autres. Ainsi, la modification, à la suite de travaux de redressement, de certains

(*) A la suite de cours et de conférences donnés au Centre d'Hydrobiologie et d'Hydrologie de l'Université de Besançon ainsi qu'à l'Ecole Nationale du Génie Rural, des Eaux et des Forêts à Paris. 
paramètres comme la pente, la vitesse d'écoulement ou la granulométrie des fonds, induit rapidement des changements qualitatifs et quantitatifs des peuplements aquatiques qui se répercutent sur le pouvoir auto-épurateur du cours d'eau et entraînent une dégradation de la qualité physicochimique de l'eau.

La richesse et la diversité des édifices biologiques, dont dépend la qualité de l'eau, sont sous la dépendance directe de la diversité des climats aquatiques, de l'hétérogénéité du milicu (mosaïques de biotopes et de biocénoses) selon les biozönotische Grundprizipien, proposés depuis longtemps déjà par Thienemann (1920-1926), dont le respect constitue le guide le plus sûr des aménagements hydrauliques et piscicoles ainsi que de la lutte contre la pollution des eaux (Verneaux, 1973; Verneaux et Leynaud, 1974).

Ce principe d'hétérogénéité (ou de diversité et de stabilité) maximale correspond par exemple au maintien, ou à la création, de tracés sinueux, de l'alternance de seuils (radiers) et de «mouilles » («profonds», «pools»), d'enrochements et des dispositifs divers permettant l'installation, la nutrition, la reproduction et la circulation des espèces vivantes.

Les pratiques actuelles, non seulement ne respectant pas mais ignorant ces données élémentaires, aboutissent à des simplifications plus ou moins accentuées des systèmes (habitats et biocénoses) et doivent être considérées, en vertu du principe logique de causalité, comme l'un des aspects du phénomène général de pollution. Ces « effets polluants», tels qu'ils ont été définis par l'un de nous (Verneaux, 1973), se manifestent de façon aiguë, parfois sur un mode catastrophique, en périodes d'étiage ou de basses eaux (niveaux du cours d'eau et de la nappe considérablement abaissés) surtout lorsque le cours d'eau sert de vecteur aux divers effluents de l'activité humaine.

\section{3 - Protocole d'étude Définition des travaux d'aménagement}

Les erreurs techniques usuelles proviennent très généralement de la méconnaissance de l'écosystème, tel qu'il vient d'être défini, dont l'étude doit être effectuée à l'échelle du bassin versant.

Les actions d'aménagement, destinées à améliorer des situations aquatiques reconnues à divers titres comme défectueuses, ne peuvent être correctement définies qu'au terme d'une diagnose écologique la plus complète possible; à cette nécessité s'oppose, sinon l'absence, du moins le caractère excessivement fragmentaire des études préalables aux actuels projets, le plus souvent réduites à quelques données hydrauliques. Les travaux qui en résultent, parfois associés à des travaux de remembrement ou d'assainissement, sont trop souvent limités à des corrections, des redressements ou des recalibrages non seulement peu satisfaisants d'un point de vue strictement hydraulique mais très généralement contraires aux bases techniques issues d'une étude rationnelle.

Les études écologiques qui nécessitent à la fois le concours d'équipes pluridisciplinaires de scientifiques de haut niveau et des délais importants (de l'ordre de trois années) peuvent être très valablement remplacées, au niveau des services d'application, par des études à caractère synthétique utili- sant un certain nombre de méthodes pratiques; une telle étude comporte successivement :

- une étude préalable du bassin versant, climatique, topographique, géologique, hydrologique, hydraulique, économique et humaine;

- une étude de la qualité physico-chimique (Nisbet et Verneaux, 1970) et biologique des eaux (Verneaux et Tuffery, 1967; Coste, 1974);

- une étude biotypologique du premier ordre (abaque morpho-dynamique et modèle ichtyologique, Verneaux, 1973; Verneaux et Leynaud, 1974) éventuellement suivie d'une analyse du deuxième ordre (invertébrés benthiques);

- enfin l'analyse au niveau de tronçons témoins stables des caractéristiques morpho-dynamiques (mosaïques de biotopes) et biologiques (mosaïques de biocénoses).

Ces éléments peuvent être réunis en l'espace de quelques mois, parfois de quelques semaines; ils permettent de déterminer les caractéristiques hydrologiques et biologiques du système, de définir son état physiologique ainsi que les appartenances typologiques de ses différents secteurs, enfin de faire la part des dispositions naturelles et des interventions de l'homme et de proposer des aménagements rationnels selon des techniques convenables en fonction de particularités écologiques clairement déterminées.

\section{4 - Les techniques d'aménagement}

La synthèse des informations de diverses natures s'effectuant au niveau de l'écologie, science des relations entre espèces et milieu, les directives en matière d'aménagement des écosystèmes proviennent nécessairement de la confrontation de plusieurs séries de données, en particulier des impératifs hydrauliques et biologiques. Issues d'un dialogue permanent, ces bases n'auraient pu être dégagées en dehors de la concordance des différents impératifs scientifiques que l'usage, cette mauvaise habitude, veut trop souvent et malencontreusement imposer.

Sans entrer dans les détails techniques, faisant par ailleurs l'objet d'une publication plus complète, nous proposons de retenir quelques éléments simples permettant d'éviter la plupart des erreurs fondamentales coutumières.

\section{CORRECTION DU LIT}

\section{A) Tracé du cours d'eau}

Les rivières à méandres apparaissant comme la forme la plus stable qu'un cours d'eau puisse prendre, en dehors des méandres imposés par des obstacles consécutifs à un manque d'entretien, il convient de conserver, ou de déterminer, un tracé sinueux (alternance de courbes et de contrecourbes) en évitant toutefois les rayons de courbure trop faibles (sièges d'une érosion intense en rive concave) ou trop forts (instables, comme les tronçons tectilignes). Comme le note Habig (1971), le tracé sinueux présente cet avantage supplémentaire sur les cours rectilignes de définir les secteurs devant être protégés.

L'aménagiste doit prendre conscience que les méandres, loin de constituer une forme dégénérée ou instable d'évolution, aboutissent au contraire à une structure hydrodyna- 
miquement stable répondant aux critères biologiques de stabilité et de diversité.

\section{B) Profils transversaux}

Après avoir rappelé que les différentes formes qu'il convient de donner aux profils transversaux sont étroitement associées aux caractéristiques du tracé en plan, il convient de remarquer que le profil trapézoïdal symétrique, malheureusement si souvent adopté pour des raisons de facilité d'exécution, n'est théoriquement admissible que pour des cours d'eau dont la différence entre niveaux de hautes eaux et de basses eaux reste très faible (phénomène peu fréquent).

Si la capacité de transport solide du cours d'eau est assez importante, celui-ci tend à reformer un lit mineur à l'intérieur de la section imposée; lorsque la pente est faible ce type de profil favorise, par sa largeur excessive, l'accumulation de dépôts sédimentaires rapidement colonisés par une végétation semi-aquatique en période de basses eaux: l'effet produit s'avérant contraire à l'effet recherché, il est préférable, pour des raisons tant biologiques qu'hydrauliques, d'adopter une section comportant lit mineur et lit majeur.

On peut choisir une section triangulaire, parabolique ou trapézoïdale selon les cas, qui permette de transiter les eaux moyennes et les étiages en maintenant des vitesses et des profondeurs en accord avec les exigences biologiques, encadrée par un lit majeur dimensionné en fonction des débits maximums et endigué si besoin est.

La conservation d'un lit mineur hétérogène correspond à la nécessité de maintenir ou d'obtenir des débits minimums compatibles avec la pérennité des édifices biologiques, les étiages constituant des périodes critiques pour l'écosystème et la qualité des eaux. Ces conditions limites sont aggravées par les pompages consécutifs à l'augmentation accrue des besoins et par la dégradation croissante de la qualité physicochimique des eaux entraînant, en période d'étiage estival, la prolifération d'organismes à cycle court, en particulier d'algues benthiques filamenteuses dont la masse constitue une forme de pollution différée pouvant entraîner des mortalités pisciaires (Loue, Port-Lesney, août 1974) ainsi que des gênes parfois considérables dans l'exploitation des ressources aquatiques (Vergon et Masson, 1973; Verneaux et Vergon, 1974).

\section{OUVRAGES DE STABILISATION ET DE PROTECTION}

\section{A) Stabilisation du lit}

Ces ouvrages sont non seulement destinés à réduire l'énergie cinétique qui, liée à de fortes pentes, augmente la puissance érosive du cours d'eau, mais aussi à maintenir un niveau d'étiage et une hétérogénéité favorables aux organismes aquatiques. Il convient de noter que les travaux de rectification mal conçus tendent au contraire à diminuer la rugosité des supports (homogénéisation) et les pertes de charge (régularisation, raccourcissement...) et corrélativement à augmenter les vitesses d'écoulement et la puissance érosive... ainsi que les dépôts sédimentaires en aval.

L'aménagement du lit, en particulier des tronçons rectilignes et des têtes de méandres, à l'aide de seuils rustiques, dont la fréquence et les dimensions doivent être accordées non seulement aux caractéristiques hydrodynamiques du cours d'eau mais également au tracé et aux accidents comme les ruptures de pente ou les affleurements rocheux, peut aboutir à une physiographie remarquable, stable et très favorable au développement d'un édifice biologique optimum pour le niveau typologique considéré (ex. cours supérieur de la Loue). Il est souvent préférable, pour un tronçon homogène donné (ex. Drugeon), d'implanter plusieurs seuils de faible hauteur (entre 0,5 et $1 \mathrm{~m}$ ) qu'un seuil unique de hauteur plus importante; de même le plan incliné amortisseur à granulométrie hétérogène ou une succession de cascades sont préférables à une unique chute.

Les zones turbulentes à l'aval des seuils doivent s'étendre sur une certaine longueur et la crête des ouvrages ne pas émerger en période de basses eaux; dans certains cas, un créneau central pourvu ou non d'une vanne peut s'avérer nécessaire. Lorsqu'ils sont correctement construits, ces ouvrages assurent une réoxygénation permanente de l'eau (augmentation du pouvoir auto-épurateur), constituent des habitats favorables au développement des Bryophytes, supports très hospitaliers pour la faune benthique (Thécamoebiens, Rotifères, Tardigrades, larves d'insectes aquatiques, Oligochètes... Decamps et Lafont, 1974), jouent un rôle important dans la production primaire (Dawson, 1973) et constituent des refuges à poissons précieux en période critique.

\section{B) Protection des berges}

Il convient, en ce domaine, de rechercher des solutions à la fois pérennes (ce qui n'est pas le cas des gabions) et favorables au développement des édifices biologiques (ce qui n'est pas le cas des parois bétonnées).

Une solution satisfaisante consiste à protéger les rives concaves à l'aide d'enrochements convenablement dimensionnés et disposés, plantés de boutures de saule buissonnant (ex. Salix purpurea) dont les rameaux demeurent de faible diamètre. Les plantations des digues et de la partie supérieure des berges assurent non seulement la protection et la fixation des sols mais contribuent également à l'épuration des apports latéraux (eaux de ruissellement chargées de substances fertilisantes) et constituent des abris pour les imagos (adultes aériens) de très nombreux insectes à larves aquatiques.

Les mêmes données peuvent être utilisées pour la réalisation d'épis latéraux en enrochements qui, augmentant l'hétérogénéité des mosaïques, constituent des dispositifs biologiquement favorables.

\section{5 - Conclusion}

Nous avons tenté dans cette note préliminaire de montrer qu'une conception rationnelle des aménagements de cours d'eau, loin d'être hasardeuse ou réduite à une technique «rhéologique» sommaire et universelle, apparaît comme le fruit, la résultante pratique, d'une démarche écologique considérée comme la phase ultime d'une analyse rationnelle des situations.

Il convient de souligner que l'écologie, contrairement à une opinion coutumière, n'est pas davantage l'apanage du biologiste que du chimiste, de l'hydraulicien ou du géographe mais, science de synthèse en voie d'élaboration, s'exprime au niveau de chaque contribution par un sens aigu du relatif et de la recherche d'un essentiel commun.

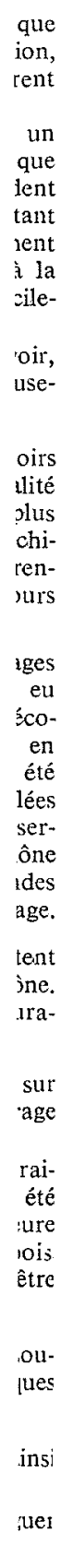

bit

ste

ior

au

ac

ise

Ire
di

de 
Ne pas ignorer, ne pas négliger la compréhension des disciplines, constitue la démarche nécessaire à tout progrès en ce domaine.

L'occasion nous ayant été donnée, au cours de l'examen de plusieurs projets d'aménagement, de confronter les exigences hydrauliques et biologiques, nous avons pu constater leur très logique concordance lorsque le technicien veut bien ne pas confondre les lois de l'hydraulique des canaux à fond rigide et celles de l'hydraulique fluviale.

Les quelques principes et données simples précédemment exposés doivent être considérés comme une première démarche vers une connaissance écologique plus complète qui, pour résoudre les problèmes de plus en plus aigus posés par la détérioration accélérée des ressources naturelles, nécessite, par un effort accru de recherche et d'imagination, l'abandon des solutions de facilité trop partielles, souvent aléatoires et trop généralement nuisibles.

\section{Auteurs cités}

Coste (M.). - «Etudes sur la mise au point d'une méthode bio'ogique de détermination de la gualité des eaux en milieu fluvial " (1974). C.T.G.R.E.F., Div. Qual. Eaux P. Pisc. Paris : $80 \mathrm{p}$.

DAwson (F. H.). - Notes on the production of stream Bryophytes in the high Pyrenees (France) (1973). Annls Limnol, 9 (3): 231-240.
Decamps (H.) et LAFONT (M.). - Cycles vitaux et production des Micrajema pyrénéennes dans les mousses d'eau courante ('Trichoptera, Brachycentridae) (1974). Annls. Limnol, 10 (1) 1-32.

HABIG (H.). - «La pratique de travaux d'hydraulique filuviale» (1971). Centre Géogr. appl., Univ. Strasbourg : ronéo.

Nisbet (M.) et Vernenux (J.). - Composantes chimiques des eaux courantes. Discussion et proposition de classes en tant que bases d'interprétation des ana'yses chimiques (1970). Annls. Limnol., 6 (2): 161-190.

Thienemann (A.). - Die Grundlagen der Biozönotik und Monards faunistische Prinzipien (1920). Festch. Zschokke, 1 (4): 1-14.

Thienemann (A.). - Die Binnengewässer Mitteleuropas. Eine limnologische Einführung (1925). Die Binnegew, 1: $255 \mathrm{p}$.

VERgON (J.-P.) et MASSON (J.-P.). - «Sur le développement intense des algues filamenteuses dans le bassin du Doubs en 1972 ». Comm. $2^{\circ}$ Congrès Poll. et Protec. Eaux, Inst. Pasteur Lyon, 5-6-7 déc. : 16 p. (1972).

Verneaux (J.). - "Recherches éco'ogiques sur le réseau hydrographique du Doubs. Esssai de biotypo'ogie " (1973). Thèse Doctorat ès Sci., Biol. Anim., Fac. Sci. Univ. Besançon : 300 p.

Verneaux (J.) et Leynaud (G.), - - Introduction à la définition d'«objectifs » puis de "critères» de la qualité des eaux courantes. Démarches, principes et orientations générales : (1974). C.T.G.R.E.F., Div. Qual. Eaux P. Pisc:, Paris: 28 p:

Verneaux (J.) et Tuffery (G.). - Une méthode zoologique pratiquo de détermination de la qualité biologique des eaux courantes. Indices biotiques (1967). Ann. Sci. Univ. Besançon, Zool., 3: 79-90.

Verneaux (J.) et Vergon (J.-P.). - A propos d'un processus " d'auto-épuration » des cours d'eau par les algues benthiques filamenteuses (1974). 13 ${ }^{\mathrm{e}}$ J. Hydraul., Soc. Hydrotec. Fr. $6(1): 7 \mathrm{p}$.

\section{Discussion}

Président : M. G. Leynaud

M. le Président remercie vivement $M$. Vernenux qui a bien montré que l'éco'ogie, loin d'être le monopole de quelques spécialités, est une science de synthèse qui fait appel à toutes les discip'ines.

Il onvre ensuite la discussion en espérant que de nombreux auditeurs voudront profiter de la présence de $M$. Verneaux pour obtenir la réponse à bien des questions techniques qui lui sont fami'ières.

M. Marchal (Service de la Navigation, Strasbourg) pose au conférencier la question suivante

Les travaux actuellement en cours d'étude pour l'aménagement par canalisation de la va'lée du Doubs, dans le cadre de la liaison Rhin-Rhône, vont entrấner de profondes modifications écologiques; peut-on, à la lumière des transformations observées notamment par les chercheurs de l'Université de Besancon - entre 1800 et 1830 du fait de la première canalisation du Doubs, conner quelques renseignements qui pourront être utiles pour conserver un équilibre écologique satisfaisant lors de la nouvelle canalisation ?

\section{Verneaux répond :}

Bien que ma thèse de Doctorat d'Etat ait essentiellement porté sur la recherche d'une biotypologie du réseau hydrologique franc-comtois, ce qui m'a donné l'occasion d'étudier l'écologie de cette région, mon Service n'a pas reçu de demande d'étude sur le projet de nouvelle canalisation du Doubs.

Je ne puis donc vous donner qu'un point de vue personnel sur ce projet. Je suis opposé à la liaison envisagée - qui joindrait la mer du Nord à la Méditerranée en empruntant la vallée du Doubs puis la Saône - à la fois pour des raison écologiques et pour des raisons économiques.
L'écosystème «canal» est bien différent de l'écosystème «cours d'eau»; il s'apparenterait plutôt à celui d'un étang à niveau variable:

En ce qui concerne l'économie, je suis inquet du fait que près des deux tiers de la population du bassin sont groupés le long de la vallée du Doubs et que le canal envisagé renforce le caractère monoaxial de l'aménagement régional.

D'autre part, ce canal fait peser un grave danger sur l'alimentation en eau de cette région karstique qui ne "récupère » ses eaux qu'au niveau de la vallée du Doubs; or, le drainage d'une grande partie de ces eaux, et notamment celles des nombreuses émergences du karst qui s'échelonnent le long de cette vallée, s'oppose à un programme cohérent de préservation de ces ressources.

D'autres raisons me font penser qu'il serait plus économique de substituer au tracé envisagé dans la vallée du Doubs, un tracé p'us court passant à travers le département de la Haute-Saône..., ce qui n'est peut-être pas plus difficile à réaliser.

Je vous donne là un point de vue d'écologiste et, je le répète, ün point de vue strictement personnel.

Je ne pense pas, observe M. Marchal, que ce soit ici le lieu de discuter du problème généra! de la canalisation des rivières; une longue expérience s'étendant dans le monde entier ne permet pas de condamner d'une façon définitive ce type d'aménagement.

Vous avez montré, dans votre exposé, que l'écologie devait permettre de prévoir comment se produisent un certain nombre d'équilibres. C'est pourquoi je vous ai demandé de quelle manière les équilibres dans la vallée du Doubs avaient été modifiés par la promière canalisation de cette rivière. Je pense qu'une étude de ce genre pourrait utilement servir de point de départ à un nouvel 
examen du projet... Ne croyez pas que les hydrauliciens et les techniciens des voies navigables se désintéressent de la biologie des fleuves et cours d'eau qu'i's ont à gérer.

Cette biologie - je le reconnais - est différente de celle du fleuve naturel, mais elle n'est pas forcément plus mauvaise. Il pourrait exister des moyens d'améliorer les choses à partir des leçcns tirées des recherches déjà faites dans le domaine écologique. C'est d'ailleurs ce qui est envisagé dès que la prise en considération du projet sera réalisée.

J'ai effectué, précise M. Verneaux, des études de qualité d'eau, aux mêmes niveaux, dans des tronçons situés en rivière canalisée et en rivière subnaturel'e (il n'y a en l'occurrence pas de rivières naturelles). La qualité de l'eau dans les tronçons canalisés est inférieure de moitié à celle de l'eau du cours d'eau, et ceci pour des raisons bien connues.

En effet, la qualité de l'eau est sous la dépendance étroite des caractéristiques, de l'hétérogénéité du milieu qui entraîne celle de l'édifice biologique. Ce n'est que grâce à l'existence d'un édifice biologique complexe que l'eau peut être nature'lement épurée par les systèmes aquatiques.

Le canal, au contraire, correspond à une homogénéisation très efficace des paramètres de l'environnement. C'est une simplification énorme de l'édifice biologique, et cela se traduit par un abaissement considérable du pouvoir auto-épurateur des cours d'eauh

J'ajoute à ce'a que le canal envisagé est branché sur le système alsacien et rhénan. Connaissant la qualité des eaux du canal d'Alsace et celles du Rhin, j'émets les craintes les plus vives quant à la qualité des eaux de ce canal; ces eaux ne pourront, en aucune façon, être auto-épurées par le canal lui-même et le projet me semble incompatible avec celui actuellement en cours, visant à rétablir une qualité d'eau acceptab'e (projet "Doubs-rivière propre »).

M. MoRIN (SOGREAH) aimerait voir préciser les raisons qui ren-dent les «crues débordantes» dangereuses pour l'écologie.

C'est, dit M. Verneaux, qu'il n'est pas normal qu'un système aquatique (pas plus qu'un individu) présente des crises aiguës comme une crue dévastatrice.

Lorsque le débit de la crue s'écoule en entier dans le lit mineur du cours d'eau, on assiste à un arrachement des espèces animales, ainsi qu'à une modification des substrats. Lorque le cours d'eau déborde, un grand nombre d'espèces, en particulier les poissons, suivent le mouvement de l'eau et finissent au milieu des prés. Dans nombre de bassins, lorsque cela se produit, les gardes-pêche ont bien du mal à éviter les ramassages intempestifs de poissons notamment de perches ou de brochets - qui demeurent captifs des bas-fonds du lit majeur. Ce phénomène se manifeste pour beaucoup d'espèces aquatiques.

S'il n'est pas souhaitable qu'un cours d'eau déborde, je suis, en revanche, favorable ail maintien d'un niveau d'étiage convenable car il faut qu'il y ait suffisamment d'eau dans les cours d'eau en ces périodes difficiles. A cet effet, il ne faut pas surdimensionner le lit mineur si l'on veut conserver une lame d'eau compatible avec la pérennité de l'édifice biologique.

M. Rfceveur (Agence de Bassin Loire-Bretagne) intervient en ces termes:

Vous avez été amené à réfféchir sur les conséquences bonnes sous certains aspects, dommageables pour d'autres, au plan écologique, de la technique de soutien des étiages des rivières. Quelle est votre opinion, à ce sujet et quels sont les risques à éviter à votre avis?

Je suis favorab'e d'une manière générale, répond M. Vernenux, au soutien des étiages afin d'éviter la destruction de l'édifice biologique au cours des longues périodes de sécheresse..., mais également au fait de maintenir le cours d'eau dans un lit majeur correctement défini.

Je pense à l'aménagement de la Loue. Je ne parle pas de la basse Loue, qui a été "massacrée», mais aux cours supérieur et moyen, aménagés il y a plusieurs dizaines d'années, à l'aide de barrages fixes aidant à soutenir le débit d'étiage.

Mais depuis, les pollutions mixtes chroniques de la rivière le Doubs s'opposant à l'utilisation de l'eau. on a été conduit pour alimenter Besançon - notamment - à prendre de l'eau dans la rivière la Loue. On a commencé par en prendre un peu.... puis un peu p'us..., puis encore plus..., puis trop. Actuellement, en période de basses eaux, les crêtes des barrages émergent et il se forme dans le bief amont une sorte de petit lac. Ceci provoque la prolifération de certaines algues et l'apparition de phénomènes de putréfaction, cela entraîne une pol'ution certaine de la rivière, à tel point que les algues bleues qui se forment sur les amas en putréfaction, lorsqu'elles sont el'es-mêmes en voie de décomposition, libèrent des produits toxiques létaux pour les poissons.

J'ai pu ainsi constater la mort de $800 \mathrm{~kg}$ de truites dans un secteur où il n'y avait pas d'autre source manifeste de pollution que les algues bleues et la présence de vase réductrice. Cet accident était dû essentiellement au prólèvement d'un débit trop important d'eau pour faire face aux besoins de l'alimentation, ce prélèvement aboutissant à un assèchement de la crête des barrages fixes et à la formation d'un plan d'eau stagnante en amont, supportant difficilement une augmentation de la température.

Dans les conditions actuelles d'exploitation, il faudrait prévoir, l'entretien des chenaux centraux par l'action de vannes judicieusement disposées.

M. THÉvENIN évoque l'effet des lâchures des barrages-réservoirs construits en tête des val'ées et qui libèrent des eaux dont la qualité est souvent très différente de celle des eaux amont; elles sont plus froides en été, généralement désoxygénées, et leur composition chimique a été modifiée pendant la période de stockage. Le conférencier connait-il les effets de ces lâchures sur l'écologie du cours d'eau en aval de l'ouvrage?

Ce cas est sans doute très différent de celui des petits barrages fixes dont $j$ 'ai déjà parlé, répond M. Verneaux; n'ayant pas eu loccasion de l'étudier, je n'ai guère d'opinion sur l'influence écologique de ce type d'ouvrage. Le soutien des étiages me paraît, en principe, bénéfique à condition que les lâchures effectuées en été n'entraînent pas les limons et les matières fermentescibles accumulées sur les fonds. M. Verneaux résume, à titre d'illustration, ses observations sur les !âchures de certains barrages implantés sur le Rhône dès sa sortie de Suisse : les vases noires ou brunâtres nauséabondes asphyxient le lit sur un long secteur du Rhône à l'aval du barrage.

M. THÉVENIN demande au conférencier de quelle époque datent les constatations qu'i! a effectuées en aval des barrages du Rhône. Il signale que la Ville de Genève a mis en service une station d'épuration qui devait améliorer la situation à ce point de vue.

Mes observations datent de cette année mais portent, non sur les lâchures de barrage du Verbois, mais sur ce'les d'un barrage implanté sur l'Isère, répond M. VernEAuX.

A Verbois, la situation s'est, en effet, amélio:ée depuis le traitement partiel des eaux usées de la vil'e de Genève; cela m’a été confirmé par les pêcheurs professionnels de la région. A l'heure actuelle, l'indice biotique moyen, à l'aval de la retenue de Verbois, n'est que de 3 à 4 sur 10 ; mais il y a deux ans, il devait être encore plus faible.

M. RECEVEUR revient sur la question des barrages destinés à soutenir les étiages des cours d'eau et sur les conséquences éco'ogiques de ce type de lâchures.

M. ROUYER (Ingénieur en Chef du GREF, Poitiers) formu'e ainsi son opinion sur ce point:

En ce qui concerne les lacs de barrage, il convient de distinguer entre:

- les vidanges totales de la retenue;

- les lâchures prises à un certain niveau pour relever les débits d'étiage.

Dans le premier cas, i! y a toujours des conséquences néfastes pour l'aval

Dans le deuxième cas, les conséquences devraient être a priori bénéfiques.

Pour M. Verneaux, il faut éviter d'apporter d'une part des eaux de fond entrainant des "fines » et, d'autre part, des eaux de surface trop réchauffées et trop riches en phyto-plancton; il faut s'efforcer d'organiser le système de soutirage de manière à ce que les lâchures proviennent d'une tranche moyenne - judicieusement choisie - du réservoir.

M. RÉMÉNIÉRAs rappelle que certains réseryoirs alimentant des réseaux d'irrigation (ou d'addluction d'eau) comportent des tours de prises d'eau étudiées pour permettre le prélèvement de l'eau ì divers niveaux selon la saison.

M. Lefoulon (Inspecteur Général, EDF) intervient en ces termes: II vient d'être question des inconvénients écologiques des lâchures des barrages, présentées sous forme de gros débits intermittents d'une cau très froide. 
Je tiens à faire remarquer que les barrages-réservoirs, qui doivent être exploités par éc'usées, ont été complétés à leur aval par la création de bassins de compensation d'une capacité convenable et de faible profondeur. Grâce à ces bassins, les lâchures sont étalécs sur vingt-quatre heures. Les cours d'eau reçoivent donc un débit normal et d'une température ambiante, l'eau s'étant réchauffée dans les bassins de faible profondeur.

Au point de vue écologique, lorsque le bilan d'une opération est largement positif, il convient de la réaliser. Je citerai les barragesréservoirs créés sur la Seine et ses affluents cn amont de la région parisienne. Leur exploitation judicieuse permettra, à la fois, l'écrêtement des crues et le relèvement des débits d'étiage pour le plus grand bicn des populations riveraines.

M. Verneaux insiste sur le fait que l'écologie est une science de synthese:

Dans un raisonnement écologique, il faut tenir compte, dit-il, des multiples nécessités humaines, sociales, économiques du pays et dc la région intéressée. On n'a pas le droit d'opposer (c'est une vieille idée que je combats parce qu'clle est fausse) l'écologiste au sociologue, à l'économiste, au physicien, à l'hydraulicien; c'est, au contraire, la réunion de ces différents points de vue, à partir de bases scientifiques et techniques, oui peut permettre de choisir la «so'ution écologique».
On ne devient écologiste que par un raisonnement synthétique a partir de la confrontation de tous les points de vue. C'est pourquol je prétends que l'on ne peut penser «écologie» qu'en fonction des exigences de l'homme. dans les conditions de son développement.

M. le Président revient sur la température de l'eau restituée par les barrages-réservoirs :

Dans certains bassins, en particulier sur la Dordogne, dit-il, les bassins de compensation sont notoirement insuffisants; il en résulte des «ondes de lâcher» très prononcées. En outre, on injecte ainsi brusquement des eaux relativement froides dans un secteur qui, écologiquement, correspond à une température de l'eau plus éevée.

Dans ces conditions, on ne reconstitue pas un bon cours d'eau truites; du fait des variations de débit et de température correspondant aux lâchures, on passe d'un bon cours d'eau à truites à un mauvais cours d'eau à cyprinidés.

M. VERnEAux est bien d'accord sur ce point; à titre d'illustration, il résume des observations du même genre qu'il a faites sur l'Ardèche (déversement d'eaux froides du bassin de la Loire par les «aménagements » de Montpezat) et sur l'Ain, à l'aval du barrage de Vouglans.

En l'absence de nouvelles interventions, M. le Président clôt la dioussion et lève la séance en remerciant tous ceux qui ont contribué à l'animer. 